\title{
Chaotic behavior in real dynamics and singular values of family of generalized generating function of Apostol- Genocchi numbers
}

\author{
Mohammad Sajid \\ College of Engineering, Qassim University, Buraidah, Al-Qassim, Saudi Arabia.
}

\begin{abstract}
Chaotic behavior in the real dynamics and singular values of a two-parameter family of generalized generating function of Apostol-Genocchi numbers, $f_{\lambda, a}(z)=\lambda \frac{2 z}{e^{a z}+1}, \lambda, a \in \mathbb{R} \backslash\{0\}$, are investigated. The real fixed points of $f_{\lambda, a}(z)$ and their nature are studied. It is seen that bifurcation and chaos occur in the real dynamics of $f_{\lambda, a}(z)$. It is also found that the function $f_{\lambda, a}(z)$ has infinitely many singular values for $a>0$ and $a<0$. The critical values of $f_{\lambda, a}(z)$ lie inside the open disk, the annulus and exterior of the open disk at center origin for $a>0$ and $a<0$.
\end{abstract}

Keywords: Fixed points, critical values, singular values, bifurcation, chaos, Lyapunov exponents.

2010 MSC: 30D05, 37C25, 37M25, 58K05.

(C)2019 All rights reserved.

\section{Introduction}

For describing properties of Julia sets and Fatou sets with other studies in the real and complex dynamics, investigations on fixed points and singular values of functions are of great importance. The dynamical properties of entire or meromorphic functions with infinitely many singular values are less studied in comparison to that of functions with finitely many singular $[6,11,13,14,22]$. The singular values of the one-parameter family of generalized generating function of Bernoulli's numbers are computed in [20] and its reverse family are discovered in [19]. The singular values of transcendental meromorphic functions are discussed by Zheng [25]. The theory of dynamics of transcendental functions is given in [2]. The exploration of dynamics of a real function has become an important topic, partially due to the fact that it deduce the iterations of the function in the complex plane which is mainly influenced by its real dynamics $[6,12,16,23]$. The real dynamics of the cubic polynomials, generalized logistic maps and oneparameter family of transcendental functions are given in $[1,10,15,21]$ respectively. The real fixed points are described for one-parameter family of function $\frac{x}{b^{x}-1}$ in [17] and for a two-parameter family $\lambda\left(\frac{x}{b^{x}-1}\right)^{n}$ in [8]. The study of some recent chaotic systems can be seen in [3, 4, 24].

A point $z^{*}$ is said to be a critical point of $f(z)$ if $f^{\prime}\left(z^{*}\right)=0$. The value $f\left(z^{*}\right)$ corresponding to a critical point $z^{*}$ is called a critical value of $f(z)$. A point $w \in \hat{\mathbb{C}}$ (extended complex plane) is said to be an

Email address: msajd@qu.edu.sa (Mohammad Sajid)

doi: $10.22436 /$ jmcs.019.01.06

Received: 2018-11-19 Revised: 2019-02-14 Accepted: 2019-02-20 
asymptotic value for $f(z)$, if there exists a continuous curve $\gamma:[0, \infty) \rightarrow \hat{\mathbb{C}}$ satisfying $\lim _{t \rightarrow \infty} \gamma(t)=\infty$ and $\lim _{t \rightarrow \infty} f(\gamma(t))=w$. A singular value of $f$ is defined to be either a critical value or an asymptotic value of $f$. A function $f$ is called critically bounded or function of bounded type if the set of all singular values of $f$ is bounded, otherwise it is said to be function of unbounded type. A point $x$ is called a fixed point of function $f(x)$ if $f(x)=x$. A fixed point $x_{f}$ is said to be an attracting, neutral (indifferent) or repelling if $\left|f^{\prime}\left(x_{f}\right)\right|<1,\left|f^{\prime}\left(x_{f}\right)\right|=1$ or $\left|f^{\prime}\left(x_{f}\right)\right|>1$, respectively. The Lyapunov exponent of the function $f(x)$, for a given trajectory $\left\{x_{k}: k=0,1,2, \ldots\right\}$ starting with an initial point $x_{0}$, is defined as

$$
\mathrm{L}=\lim _{\mathrm{k} \rightarrow \infty} \frac{1}{\mathrm{k}} \sum_{i=0}^{\mathrm{k}-1} \ln \left|\mathrm{f}^{\prime}\left(\mathrm{x}_{\mathrm{i}}\right)\right| .
$$

It is well known that the behavior of a dynamical system is chaotic if the Lyapunov exponent of the function $f(x)$ is a positive number [7].

Let

$$
\mathcal{F}=\left\{f_{\lambda, a}(z)=\lambda \frac{2 z}{e^{a z}+1}: \lambda, a \in \mathbb{R} \backslash\{0\}, z \in \mathbb{C}\right\}
$$

be two-parameter family of meromorphic transcendental functions. The function $f_{\lambda, a} \in \mathcal{F}$ is neither odd nor even as well as not periodic. This family $\mathcal{F}$ is associated to the generalized Apostol-Genocchi numbers and Apostol-Genocchi polynomial with parameters $a>0$ and $b>0$ which is found in [5] as

$$
\frac{2 t}{\lambda b^{t}+a^{t}}=\sum_{n=0}^{\infty} G_{n}(a, b ; \lambda) \frac{t^{n}}{n !} .
$$

For $\lambda=1, a=1, b=e^{a}, t=z$, the left hand side function in this relation becomes function of our family $\mathcal{F}$.

This paper is a generalization of work [9] of the family of functions $\lambda \frac{2 z}{e^{z}+1}$ which is a generating function of the Genocchi numbers. The paper [9] contains almost similar results and proofs from [18, 21]. Besides we provide simpler proof of the result on the number of fixed points. The following is the organization of our paper: the real fixed points of $f_{\lambda, a} \in \mathcal{F}$ as well as their nature are given in Section 2. In Section 3, the bifurcation and chaos in the real dynamics are shown graphically. It is explored that the function $f_{\lambda, a} \in \mathcal{F}$ has infinitely many singular values in Section 4. Moreover, it is found that the critical values of $f_{\lambda, a}(z)$ lie inside open disks and the annulus at center origin for $a>0$ as well as $a<0$.

\section{Real fixed points of $f_{\lambda, a} \in \mathcal{F}$ and their nature}

The existence and nature of the real fixed points of the function $f_{\lambda, a} \in \mathcal{F}$ are described in the present section. The following theorem gives that the function $f_{\lambda, a}(x)$ has two real fixed points.

Theorem 2.1. Let $\mathrm{f}_{\lambda, \mathrm{a}} \in \mathcal{F}$. Then, the function $\mathrm{f}_{\lambda, \mathrm{a}}(\mathrm{x})$ has one fixed point 0 for all $\lambda$, one nonzero real fixed point $x_{\lambda}$ for $\lambda>\frac{1}{2}$, and $f_{\lambda, a}(x)$ has no nonzero real fixed points for $\lambda \leqslant \frac{1}{2}$. For $\lambda>1$, if $a>0$, then the fixed point $x_{\lambda}$ of $\mathrm{f}_{\lambda, \mathrm{a}}(\mathrm{x})$ is positive and if $\mathrm{a}<0$, then $\mathrm{x}_{\lambda}$ is negative. For $\frac{1}{2}<\lambda<1$, if $\mathrm{a}>0$, then the fixed point $\mathrm{x}_{\lambda}$ of $\mathrm{f}_{\lambda, \mathrm{a}}(\mathrm{x})$ is negative and if $\mathrm{a}<0$, then $\mathrm{x}_{\lambda}$ is positive.

Proof. To find fixed points, we solve the equation $f_{\lambda, a}(x)=x$. Then, this equation has two real solutions 0 for all $\lambda$ and $x_{\lambda}=\frac{1}{a} \ln (2 \lambda-1)$ for $\lambda>\frac{1}{2}$. There is no real nonzero solutions for $\lambda \leqslant \frac{1}{2}$. Hence, the function $f_{\lambda, a}(x)$ has a fixed point 0 for all $\lambda$ and other real nonzero fixed point $x_{\lambda}$ for $\lambda>\frac{1}{2}$. It is easily observed that, for $\lambda>1$, the fixed point $x_{\lambda}$ of $f_{\lambda, a}(x)$ is positive if $a>0$ and negative if $a<0$. For $\frac{1}{2}<\lambda<1$, the fixed point $x_{\lambda}$ of $f_{\lambda, a}(x)$ is negative if $a>0$ and $x_{\lambda}$ is positive if $a<0$.

Remark 2.2. The proof of Theorem 2.1 is simpler and shorter than the proof of a similar result found in [9] for $a=1$.

Lemma 2.3. Let $\phi(x)=x f_{a}^{\prime}(x)+f_{a}(x)$, where $f_{a}(x)=\frac{2 x}{e^{a x}+1}$. Then 
(i) for $a>0$,

$$
\phi(x)=\frac{2 x}{\left(e^{a x}+1\right)^{2}}\left[(2-a x) e^{a x}+2\right] \begin{cases}<0, & \text { for } x<0, \\ =0, & \text { for } x=0, \\ >0, & \text { for } 0<x<x_{1}^{*} \\ =0, & \text { for } x=x_{1}^{*} \\ <0, & \text { for } x>x_{1}^{*},\end{cases}
$$

where $x_{1}^{*}$ is the unique positive real root of the equation $(2-\mathrm{ax}) e^{\mathrm{ax}}+2=0$;

(ii) for $\mathrm{a}<0$,

$$
\phi(x)=\frac{2 x}{\left(e^{a x}+1\right)^{2}}\left[(2-a x) e^{a x}+2\right] \begin{cases}>0, & \text { for } x<x_{2}^{*}, \\ =0, & \text { for } x=x_{2}^{*} \\ <0, & \text { for } x_{2}^{*}<x<0, \\ =0, & \text { for } x=0, \\ >0, & \text { for } x>0,\end{cases}
$$

where $x_{2}^{*}$ is the unique negative real root of the equation $(2-\mathrm{ax}) e^{\mathrm{ax}}+2=0$.

Proof. The function $\phi(x)$ is written as

$$
\phi(x)=x f_{a}^{\prime}(x)+f_{a}(x)=x \frac{2\left(e^{a x}+1-a x e^{a x}\right)}{\left(e^{a x}+1\right)^{2}}+\frac{2 x}{\left(e^{a x}+1\right)}=\frac{2 x}{\left(e^{a x}+1\right)^{2}}\left[(2-a x) e^{a x}+2\right] .
$$

Note that $\frac{x}{\left(e^{\mathbf{a x}}+1\right)^{2}}<0$ for $x<0$ and $\frac{x}{\left(e^{a x}+1\right)^{2}}>0$ for $x>0$.

(i) For $a>0$, let $q(x)=(2-a x) e^{a x}+2$, then $q^{\prime}(x)=a(1-a x) e^{a x}$ and $q^{\prime \prime}(x)=-a^{3} x e^{a x}$. It gives $\mathrm{q}^{\prime \prime}(x)<0$ for $x \in \mathbb{R}^{+}$and $\mathrm{q}^{\prime \prime}(x)>0$ for $x \in \mathbb{R}^{-}$. Therefore, the function $\mathrm{q}^{\prime}(x)$ is decreasing on $\mathbb{R}^{+}$ and increasing on $\mathbb{R}^{-}$. Since $\mathrm{q}^{\prime}(0)=\mathrm{a}, \mathrm{q}^{\prime}(\mathrm{x}) \rightarrow 0$ as $\mathrm{x} \rightarrow-\infty$ and $\mathrm{q}^{\prime}(\mathrm{x}) \rightarrow-\infty$ as $\mathrm{x} \rightarrow+\infty$, by continuity of $q^{\prime}(x)$, it follows that there is a unique $\tilde{x}_{1}>0$ such that $q^{\prime}(x)>0$ for $-\infty<x<\tilde{x}_{1}$, $\mathrm{q}^{\prime}\left(\tilde{x}_{1}\right)=0$ and $\mathrm{q}^{\prime}(x)<0$ for $x>\tilde{x}_{1}$. Hence, $q(x)$ increases in $\left(-\infty, \tilde{x}_{1}\right)$, attains its maximum at $\tilde{x}_{1}$ and decreases thereafter. Since $q(0)=4$ and $q(x) \rightarrow-\infty$ as $x \rightarrow+\infty$, then there is a unique positive $x_{1}^{*}>\tilde{x}$ such that $\mathrm{q}(\mathrm{x})>0$ for $-\infty<\mathrm{x}<\mathrm{x}_{1}^{*}, \mathrm{q}\left(\mathrm{x}_{1}^{*}\right)=0$ and $\mathrm{q}(\mathrm{x})<0$ for $\mathrm{x}>\mathrm{x}_{1}^{*}$. It follows that

$$
\phi(x)=\frac{2 x}{\left(e^{\mathrm{a} x}+1\right)^{2}} q(x) \begin{cases}<0, & \text { for } x<0, \\ =0, & \text { for } x=0, \\ >0, & \text { for } 0<x<x_{1}^{*}, \\ =0, & \text { for } x=x_{1}^{*}, \\ <0, & \text { for } x>x_{1}^{*} .\end{cases}
$$

It can be easily seen by Figure 1 (a).

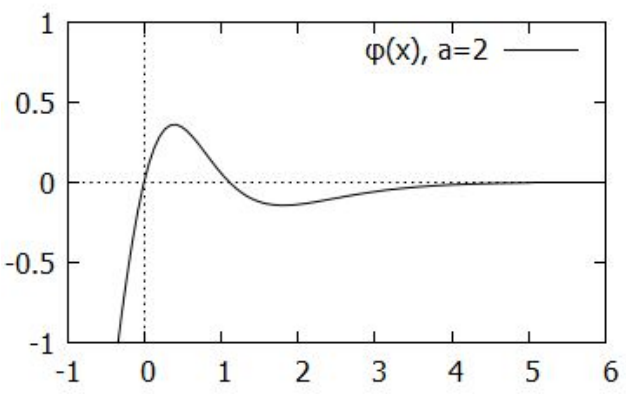

(a) For $a=2$.

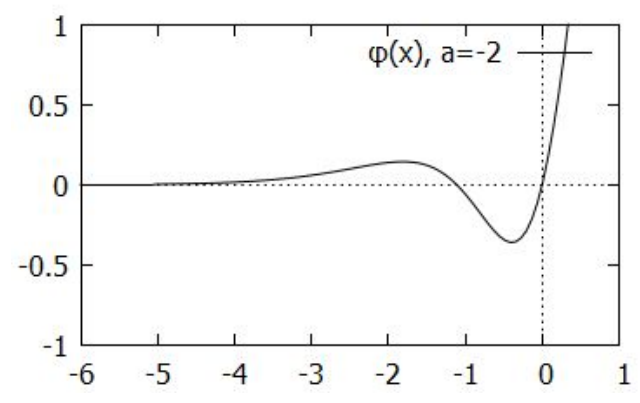

(b) For $a=-2$.

Figure 1: Graphs of $\phi(x)$ for $a>0$ and $a<0$. 
(ii) For $a<0$, let $r(x)=(2-a x) e^{a x}+2$, then $r^{\prime}(x)=a(1-a x) e^{a x}$ and $r^{\prime \prime}(x)=-a^{3} x e^{a x}$. It gives $r^{\prime \prime}(x)>0$ for $x \in \mathbb{R}^{+}$and $r^{\prime \prime}(x)<0$ for $x \in \mathbb{R}^{-}$. Therefore, the function $r^{\prime}(x)$ is increasing on $\mathbb{R}^{+}$ and decreasing on $\mathbb{R}^{-}$. Since $r^{\prime}(0)=a, r^{\prime}(x) \rightarrow+\infty$ as $x \rightarrow-\infty$ and $r^{\prime}(x) \rightarrow 0$ as $x \rightarrow+\infty$, by continuity of $r^{\prime}(x)$, it follows that there is a unique $\tilde{x}_{2}>0$ such that $r^{\prime}(x)>0$ for $-\infty<x<\tilde{x}_{2}$, $r^{\prime}\left(\tilde{x}_{2}\right)=0$ and $r^{\prime}(x)<0$ for $x>\tilde{x}_{2}$. Thus, $r(x)$ increases in $\left(-\infty, \tilde{x}_{2}\right)$, attains its maximum at $\tilde{x}_{2}$ and decreases thereafter. Since $r(0)=4$ and $r(x) \rightarrow-\infty$ as $x \rightarrow-\infty$, then there is a unique negative $x_{2}^{*}<\tilde{x}_{2}$ such that $r(x)<0$ for $x<x_{2}^{*}, r\left(x_{2}^{*}\right)=0$ and $r(x)>0$ for $x>x_{2}^{*}$. It concludes that

$$
\phi(x)=\frac{2 x}{\left(e^{a x}+1\right)^{2}} r(x) \begin{cases}>0, & \text { for } x<x_{2}^{*}, \\ =0, & \text { for } x=x_{2}^{*}, \\ <0, & \text { for } x_{2}^{*}<x<0, \\ =0, & \text { for } x=0, \\ >0, & \text { for } x>0 .\end{cases}
$$

It can be easily observed by Figure 1 (b).

Thus, it proves the lemma.

Let us define

$$
\lambda^{*}=\frac{x_{1}^{*}}{f_{a}\left(x_{1}^{*}\right)}=\frac{1}{2}\left(e^{a x_{1}^{*}}+1\right) \text { and } \lambda^{* *}=\frac{x_{2}^{*}}{f_{a}\left(x_{2}^{*}\right)}=\frac{1}{2}\left(e^{a x_{2}^{*}}+1\right) .
$$

Remark 2.4. It is easily seen that $x_{2}^{*}=-x_{1}^{*}$ and $\lambda^{* *}=\lambda^{*}$.

In the following theorem, the nature of fixed points of $f_{\lambda, a}(x)$ are shown.

Theorem 2.5. Let $\mathrm{f}_{\lambda, \mathrm{a}} \in \mathcal{F}$.

(a) The fixed point 0 of the function $\mathrm{f}_{\lambda, \mathrm{a}}(\mathrm{x})$ is attracting for $|\lambda|<1$, rationally indifferent for $|\lambda|=1$ and repelling for $|\lambda|>1$.

(b) (i) For $\mathrm{a}>0$, the fixed point $\mathrm{x}_{\lambda}$ of the function $\mathrm{f}_{\lambda, \mathrm{a}}(\mathrm{x})$ is repelling for $\frac{1}{2}<\lambda<1$, attracting for $1<\lambda<\lambda^{*}$, rationally indifferent for $\lambda=\lambda^{*}$, and repelling for $\lambda>\lambda^{*}$.

(ii) For $\mathrm{a}<0$, the fixed point $\mathrm{x}_{\lambda}$ of the function $\mathrm{f}_{\lambda, \mathrm{a}}(\mathrm{x})$ is repelling for $\frac{1}{2}<\lambda<1$, attracting for $1<\lambda<\lambda^{* *}$, rationally indifferent for $\lambda=\lambda^{* *}$, and repelling for $\lambda>\lambda^{* *}$.

Proof.

(a) Since $f_{\lambda, a}^{\prime}(0)=\lambda$, it is easy to see all cases.

(b) For $\lambda>0$ and $x \in \mathbb{R}$, it is easily seen that $f_{\lambda, a}(x)>0$ for $x>0$ and $f_{\lambda, a}(x)<0$ for $x<0$. Since $f_{\lambda, a}^{\prime}(x)=\lambda \frac{2\left[(1-a x) e^{a x}+1\right]}{\left(e^{a x}+1\right)^{2}}$ and $x_{\lambda}$ is a fixed point of $f_{\lambda, a}(x)$, then

$$
f_{\lambda, a}^{\prime}(x)=\frac{1}{2}\left(e^{a x_{\lambda}}+1\right) \frac{2\left[\left(1-a x_{\lambda}\right) e^{a x_{\lambda}}+1\right]}{\left(e^{a x_{\lambda}}+1\right)^{2}}=1-\frac{a x_{\lambda}}{1+e^{-a x_{\lambda}}} .
$$

(i) For $a>0$, the function $\frac{x}{f_{a}(x)}$ is increasing on $\mathbb{R}$ since $\left(\frac{x}{f_{a}(x)}\right)^{\prime}=\frac{1}{2} a e^{a x}>0, x \in \mathbb{R}$.

For $\frac{1}{2}<\lambda<\lambda^{*}$, this case is divided into two parts since the fixed point $x_{\lambda}$ of $f_{\lambda, a}(x)$ is negative for $\frac{1}{2}<\lambda<1$ and positive for $\lambda>1$.

For $\frac{1}{2}<\lambda<1$, by Equation (2.1), $f_{\lambda, a}^{\prime}(x)>1$ since $x_{\lambda}<0$. Therefore, the fixed point $x_{\lambda}$ of $f_{\lambda, a}(x)$ is repelling fixed point for $\frac{1}{2}<\lambda<1$.

For $1<\lambda<\lambda^{*}$, since the function $\frac{x}{f_{a}(x)}$ is increasing on $\mathbb{R}^{+}$and $\lambda=\frac{x_{\lambda}}{f_{a}\left(x_{\lambda}\right)}$, so $\frac{x_{\lambda}}{f_{a}\left(x_{\lambda}\right)}<\frac{x_{1}^{*}}{f_{a}\left(x_{1}^{*}\right)}$. It gives $e^{a x_{\lambda}}<e^{a x_{1}^{*}}$. Hence, $x_{\lambda}<x_{1}^{*}$. By Lemma $2.3(i), \phi\left(x_{\lambda}\right)>0$. Since $f_{\lambda, a}^{\prime}\left(x_{\lambda}\right)=\frac{\phi\left(x_{\lambda}\right)}{f_{a}\left(x_{\lambda}\right)}-1$, then $f_{\lambda, a}^{\prime}\left(x_{\lambda}\right)+1=\frac{\phi\left(x_{\lambda}\right)}{f_{a}\left(x_{\lambda}\right)}>0$. It follows that $\left|f_{\lambda, a}^{\prime}\left(x_{\lambda}\right)\right|<1$. Thus, the fixed point $x_{\lambda}$ of $f_{\lambda, a}(x)$ is attracting for $1<\lambda<\lambda^{*}$. 
For $\lambda=\lambda^{*}$, it is easy to see $x_{\lambda}=x_{1}^{*}$. Now, by Lemma 2.3 (i), it follows that $f_{\lambda, a}^{\prime}\left(x_{\lambda}\right)+1=\frac{\phi\left(x_{\lambda}\right)}{f_{a}\left(x_{\lambda}\right)}=0$, which implies that $f_{\lambda^{*}, a}^{\prime}\left(x_{\lambda}\right)=-1$. Therefore, the fixed point $x_{1}^{*}$ of $f_{\lambda, a}(x)$ is rationally indifferent for $\lambda=\lambda^{*}$.

For $\lambda>\lambda^{*}$, by similar arguments used above, it follows that $x_{\lambda}>x_{1}^{*}$. Again, by Lemma 2.3 (i) and by the fact $x_{\lambda}>x_{1}^{*}$, we have $\phi\left(x_{\lambda}\right)<0$. It gives that $f_{\lambda, a}^{\prime}\left(x_{\lambda}\right)+1=\frac{\phi\left(x_{\lambda}\right)}{f_{a}\left(x_{\lambda}\right)}<0$ and hence $\left|f_{\lambda, a}^{\prime}\left(x_{\lambda}\right)\right|>1$. Therefore, $x_{\lambda}$ is a repelling fixed point of $f_{\lambda, a}(x)$ for $\lambda>\lambda^{*}$.

(ii) For $a<0$, the function $\frac{x}{f_{a}(x)}$ is decreasing on $\mathbb{R}$ since $\left(\frac{x}{f_{a}(x)}\right)^{\prime}=\frac{1}{2} a e^{a x}<0, x \in \mathbb{R}$.

For $\frac{1}{2}<\lambda<\lambda^{* *}$, this case is divided into two parts since the fixed point $x_{\lambda}$ of $f_{\lambda, a}(x)$ is positive for $\frac{1}{2}<\lambda<1$ and negative for $\lambda>1$.

For $\frac{1}{2}<\lambda<1$, Equation (2.1) gives $f_{\lambda, a}^{\prime}(x)>1$ since $x_{\lambda}>0$. It follows that the fixed point $x_{\lambda}$ of $f_{\lambda, a}(x)$ is repelling fixed point for $\frac{1}{2}<\lambda<1$.

For $1<\lambda<\lambda^{* *}$, since the function $\frac{x}{f_{a}(x)}$ is decreasing on $\mathbb{R}^{-}$and $\lambda=\frac{x_{\lambda}}{f_{a}\left(x_{\lambda}\right)}$, then $\frac{x_{\lambda}}{f_{a}\left(x_{\lambda}\right)}<\frac{x_{2}^{*}}{f_{a}\left(x_{2}^{*}\right)}$. It gives $e^{a x_{\lambda}}<e^{a x_{2}^{*}}$. Hence, $x_{\lambda}<x_{2}^{*}$. By Lemma 2.3 (ii), $\phi\left(x_{\lambda}\right)>0$. Since $f_{\lambda, a}^{\prime}\left(x_{\lambda}\right)=\frac{\phi\left(x_{\lambda}\right)}{f_{a}\left(x_{\lambda}\right)}-1$, then $f_{\lambda, a}^{\prime}\left(x_{\lambda}\right)+1=\frac{\phi\left(x_{\lambda}\right)}{f_{a}\left(x_{\lambda}\right)}>0$. It gives that $\left|f_{\lambda, a}^{\prime}\left(x_{\lambda}\right)\right|<1$. Therefore, the fixed point $x_{\lambda}$ of $f_{\lambda, a}(x)$ is attracting for $1<\lambda<\lambda^{* *}$.

For $\lambda=\lambda^{* *}$, it is easy to get $x_{\lambda}=x_{2}^{*}$. Consequently, it gives that, by Lemma 2.3 (ii), $f_{\lambda, a}^{\prime}\left(x_{\lambda}\right)+1=$ $\frac{\phi\left(x_{\lambda}\right)}{f_{a}\left(x_{\lambda}\right)}=0$ and hence $f_{\lambda^{* *}, a}^{\prime}\left(x_{\lambda}\right)=-1$. Therefore, the fixed point $x_{2}^{*}$ of $f_{\lambda, a}(x)$ is rationally indifferent for $\lambda=\lambda^{* *}$.

For $\lambda>\lambda^{* *}$, by similar arguments as above, it shows that $x_{\lambda}<x_{2}^{*}$. Again, by Lemma 2.3 (ii) and by the fact $x_{\lambda}<x_{2}^{*}$, we have $\phi\left(x_{\lambda}\right)>0$. It gives that $f_{\lambda, a}^{\prime}\left(x_{\lambda}\right)+1=\frac{\phi\left(x_{\lambda}\right)}{f_{a}\left(x_{\lambda}\right)}<0$ and hence $f_{\lambda, a}^{\prime}\left(x_{\lambda}\right)<-1$. Therefore, $x_{\lambda}$ is a repelling fixed point of $f_{\lambda, a}(x)$ for $\lambda>\lambda^{* *}$.

For $\lambda>\lambda^{*}$ and $\lambda>\lambda^{* *}$, there exist periodic points of period greater than or equal to 2 . These cases are graphically discussed in the next section.

\section{Graphical simulation of bifurcation and chaos}

In the present section, we compute and visualize dynamical behaviors of $f_{\lambda, a}(x)$ by numerical and graphical simulation. It is seen from Theorem 2.5 that the nature of the fixed point of $f_{\lambda, a}(x)$ changes when parameter $\lambda$ passes certain parameter value. If the parameter $\lambda$ increases, then, for $a>0$, the behavior of dynamical system is shown by bifurcation diagrams in Figure 2 (a) for $a=2$ and Figure 2 (b) for $a=3$. It is interesting to observe that bifurcation diagrams of $f_{\lambda, a}(x)$ display periodic doubling, periodic windows, chaotic region, etc.

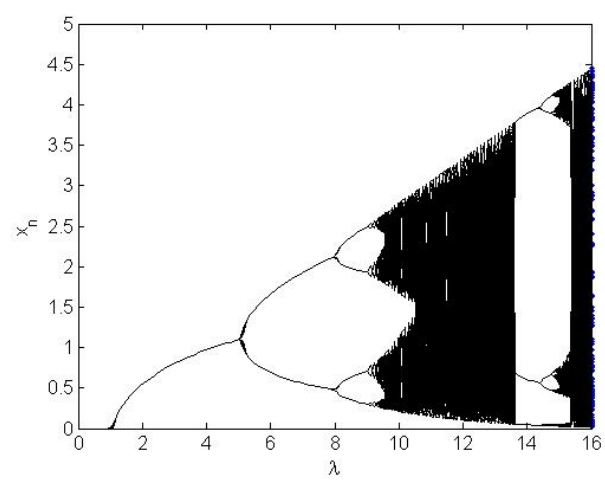

(a) For $a=2$.

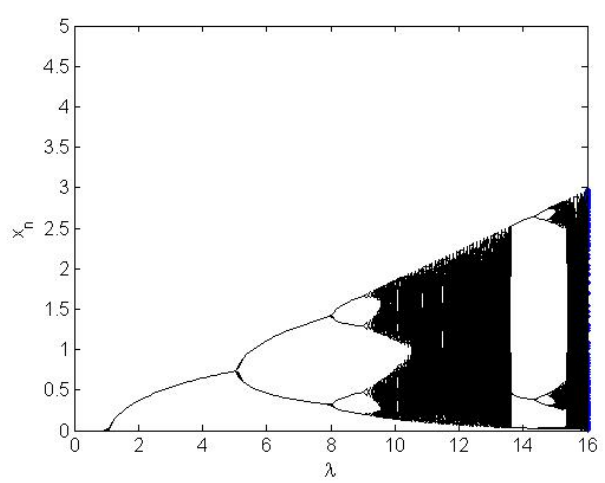

(b) For $a=3$.

Figure 2: Bifrucation diagrams for $a>0$. 
For $a<0$, the bifurcation diagrams are given in Figure 3 (a) for $a=-2$ and Figure 3 (b) for $a=-3$.

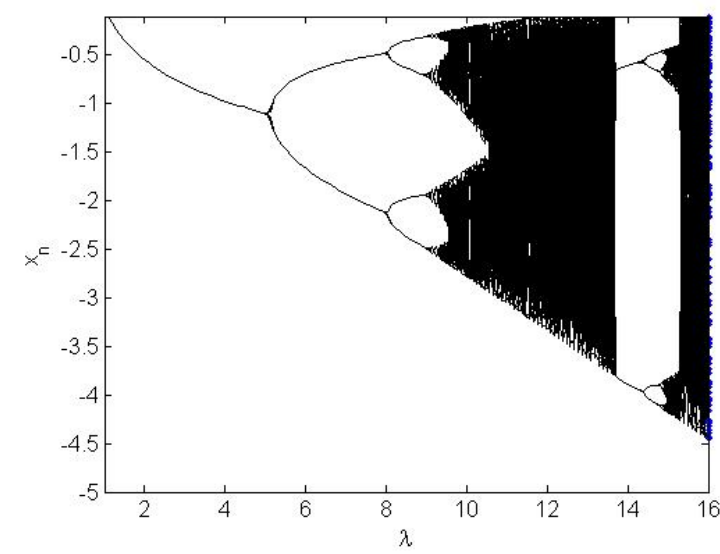

(a) For $a=-2$.

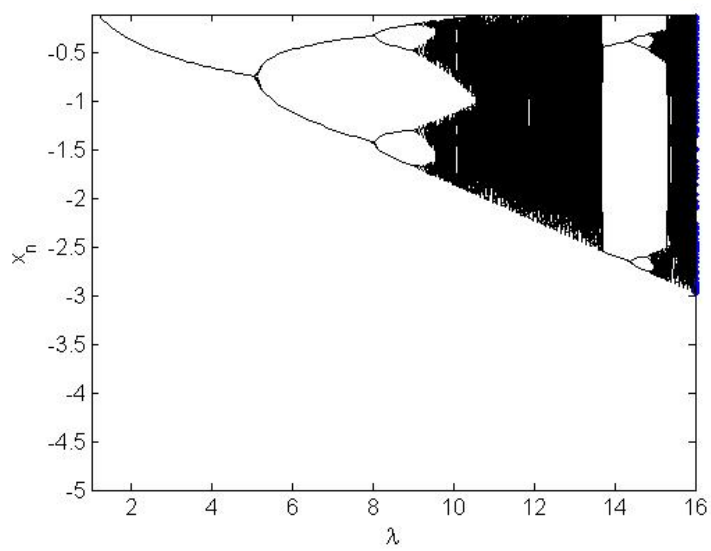

(b) For $a=-3$.

Figure 3: Bifrucation diagrams for $a<0$.

It is observed that, from Figures 2 and 3, the bifurcation diagrams $f_{\lambda, a}(x)$ are symmetric about horizontal axis for $a>0$ and $a<0$. In the real dynamics of $f_{\lambda, a}(x)$, the period doubling in the bifurcation diagram leads route to chaos. To quantify the chaos, the Lyapunov exponent is calculated. A trajectory with the positive Lyapunov exponent is chaotic provided that it is not asymptotic to an unstable periodic solution.

Using Formula (1.1), the Lyapunov exponent of the function $f_{\lambda, a}(x)$ is

$$
L=\lim _{k \rightarrow \infty} \frac{1}{k} \sum_{i=0}^{k-1} \ln \left[\lambda \frac{2\left|\left(1-a x_{i}\right) e^{a x_{i}}+1\right|}{\left(e^{a x_{i}}+1\right)^{2}}\right] .
$$

For $a>0$, the computed values of Lyapunov exponents are explored in Figures 4 (a) for $a=2$ and 4 (b) for $a=3$.

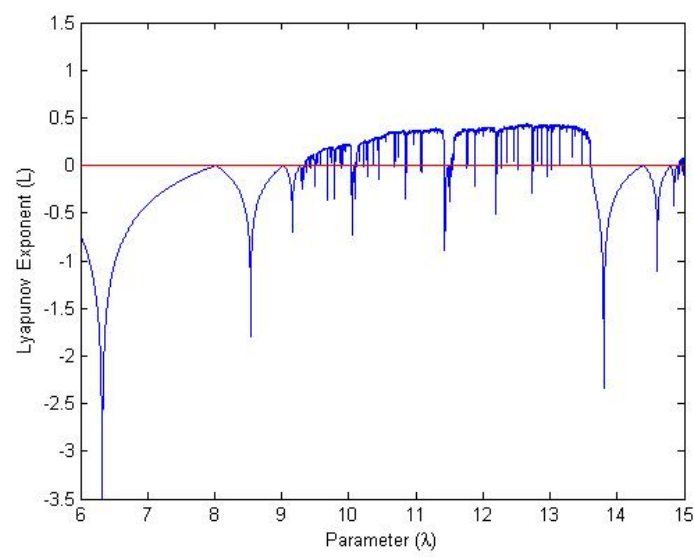

(a) For $a=2$.

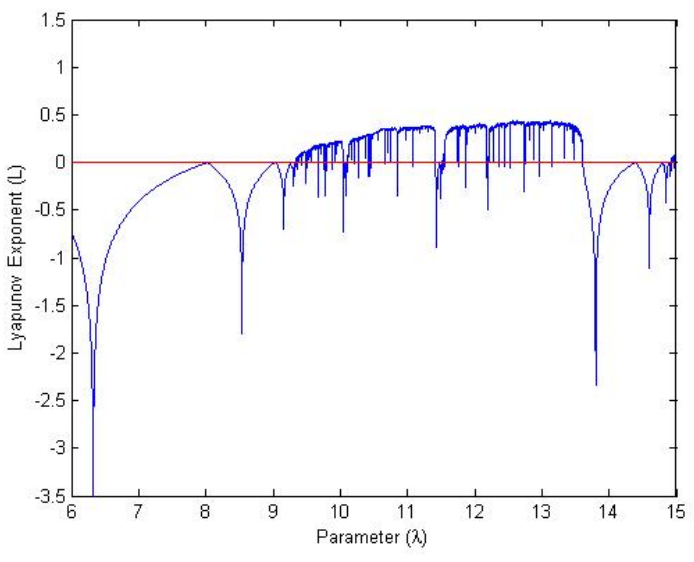

(b) For $a=3$.

Figure 4: Lyapunov exponents for $a>0$.

For $a<0$, the computed values of Lyapunov exponents are shown in Figures 5 (a) for $a=-2$ and 5 (b) for $a=-3$. 


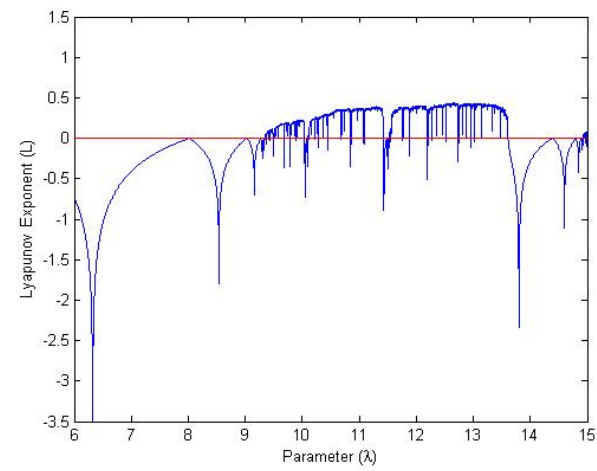

(a) For $a=-2$.

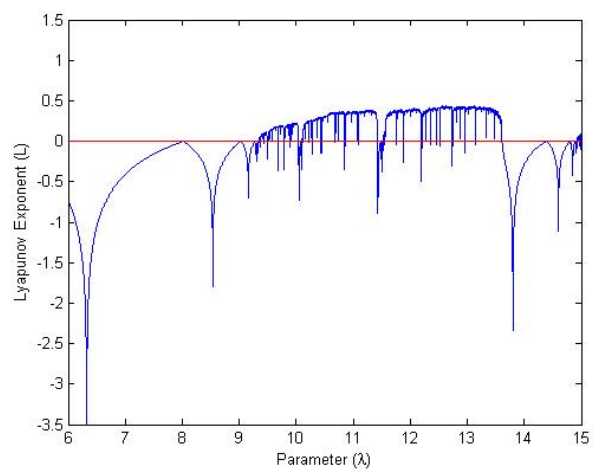

(b) For $a=-3$.

Figure 5: Lyapunov exponents for $a<0$.

From Figures 4 and 5, it is easily found that the Lyapunov exponents are positive for certain ranges of parameter $\lambda$ which exhibits the chaotic behavior in the real dynamics of $f_{\lambda, a}(x)$.

For parameter value $\lambda$ from 9.5 to 13, the bifurcation diagrams in Figures 2 and 3 and the corresponding Lyapunov exponents L in Figures 4 and 5 are represented that when Lyapunov exponents are positive, then bifurcation diagrams show dark regions which gives chaotic behavior in the real dynamics $f_{\lambda, a}(x)$. Further, between this range, the Lyapunov exponents are negative, hence it explores that chaotic region break up into non-chaotic temporary and then goes back to being chaotic.

\section{Singular values of $f_{\lambda, a} \in \mathcal{F}$}

The following theorem gives that the function $f_{\lambda, a} \in \mathcal{F}$ has infinitely many singular values:

Theorem 4.1. Let $\mathrm{f}_{\lambda, \mathrm{a}} \in \mathcal{F}$. Then, the function $\mathrm{f}_{\lambda, \mathrm{a}}(z)$ possesses infinitely many singular values.

Proof. Since $f_{\lambda, a}^{\prime}(z)=\lambda \frac{2\left[(1-a z) e^{a z}+1\right]}{\left(e^{a} z+1\right)^{2}}$, the critical points of $f_{\lambda, a}(z)$ are solutions of the equation (az1) $e^{\mathfrak{a} z}-1=0$. Let $w=a z$. Set $w=u+i v$. Separating the real and imaginary parts of $(w-1) e^{w}-1=0$, we have

$$
\begin{aligned}
\frac{v}{\sin v}+e^{v \cot v-1} & =0, \\
u & =1-v \cot v .
\end{aligned}
$$

It is observed from Figure 6 that the Equation (4.1) has infinitely many solutions since the number of intersections increases when the size of interval expands on $x$-axis. The rigorous theoretical details can be deduced from proof of [6, Proposition 1.3] for infinitely many solutions of Equation (4.1).

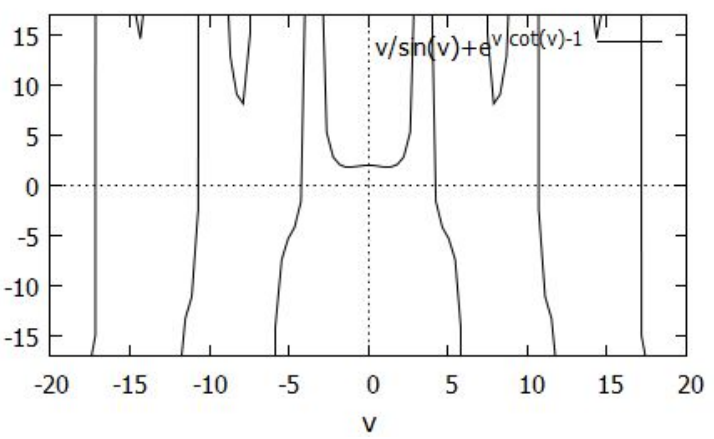

Figure 6: Graph of $\frac{v}{\sin v}+e^{v \cot v-1}$. 
Let $\left\{v_{k}\right\}_{k=-\infty, k \neq 0}^{k=\infty}$ be solutions of Equation (4.1). From Equation (4.2), $u_{k}=1-v_{k} \cot v_{k}$ for $k=$ $\pm 1, \pm 2, \pm 3, \ldots$. Then, $z_{k}=\frac{\mathfrak{u}_{k}+i v_{k}}{a}$ are critical points of $f_{\lambda, a}(z)$. The critical values $f_{\lambda, a}\left(z_{k}\right)=\lambda \frac{2 z_{k}}{e^{a} z_{k}+1}$ are distinct for $k$ nonzero integers. It follows that the function $f_{\lambda, a} \in \mathcal{F}$ has infinitely many critical values.

Since $f_{\lambda, a}(z) \rightarrow 0$ as $z \rightarrow \infty$ along positive real axis for $a>0$ and along negative real axis for $a<0$, it shows that the finite asymptotic value of $f_{\lambda, a}(z)$ is 0 . The order of growth of $f_{\lambda, a}(z)$ is finite and less than 1 , so $f_{\lambda, a}(z)$ has at most one finite asymptotic value which is 0 .

It is concluded that the function $f_{\lambda, a} \in \mathcal{F}$ has infinitely many singular values.

Let $\mathrm{H}^{+}=\{z \in \hat{\mathbb{C}}: \operatorname{Re}(z)>0\}$ and $\mathrm{H}^{-}=\{z \in \hat{\mathbb{C}}: \operatorname{Re}(z)<0\}$ be the right half plane and left half plane respectively. The following theorem shows that the function $f_{\lambda, a}^{\prime}(z)$ has no zeros in the right half plane for $a>0$ and the left half plane for $a<0$.

Theorem 4.2. Let $\mathrm{f}_{\lambda, \mathrm{a}} \in \mathcal{F}$. Then, the function $\mathrm{f}_{\lambda, \mathrm{a}}^{\prime}(z)$ has no zeros in the right half plane $\mathrm{H}^{+}$for a $>0$ except one positive point on the real axis and the left half plane $\mathrm{H}^{-}$for $\mathrm{a}<0$ except one negative point on the real axis.

Proof. Since $f_{\lambda, a}^{\prime}(z)=\lambda \frac{2\left[(1-a z) e^{a z}+1\right]}{\left(e^{a z}+1\right)^{2}}=0$, then $e^{-a z}=a z-1$. For $a>0$, let $w=a z$. Then, $e^{-w}=w-1$. Using the real and imaginary parts, we have

$$
\frac{\cos v-i \sin v}{e^{\mathfrak{u}}}=u-1+i v .
$$

When $v=0$, then $w=u>0$ and, by real part of Equation (4.3), $e^{-u}=u-1$. This equation has a positive solution.

When $v \neq 0$, then, by imaginary part of Equation (4.3), $\frac{\sin v}{v}=-e^{\mathfrak{u}}<-1$. This is not true for $v>0$ since $\left|\frac{\sin v}{v}\right|<1$. Moreover, since $\frac{\sin v}{v}$ is an even function, it is also false for $v<0$.

Consequently, the function $\mathrm{f}_{\lambda, \mathrm{a}}^{\prime}(z)$ has no zeros in $\mathrm{H}^{+}$for $\mathrm{a}>0$ except one positive point on the real axis.

Similarly, using analogous arguments as above and $z=\frac{w}{a}$ for $a<0$, the function $f_{\lambda, a}^{\prime}(z)$ has no zeros in $\mathrm{H}^{-}$except one negative point on the real axis.

Remark 4.3. $f_{\lambda, \mathrm{a}}^{\prime}(z)$ has no zeros on imaginary axis since, from Equation (4.3), $\cos v-i \sin v=1-i v$, which gives $v=0$.

The following theorems prove that the function $f_{\lambda, a} \in \mathcal{F}$ maps three different regions inside the open disk, annulus and exterior of the open disk centered at origin.

Theorem 4.4. Let $\mathrm{f}_{\lambda, \mathrm{a}} \in \mathcal{F}$ with $\mathrm{a}>0$.

(i) If $\mathrm{D}_{1}=\left\{z \in \mathbb{C}: \operatorname{Re}(z)<0\right.$ and $\left.|z|<\frac{1}{\mathrm{a}}\right\}$, then $\mathrm{f}_{\lambda, \mathrm{a}}\left(\mathrm{D}_{1}\right) \subset\left\{w \in \mathbb{C}:|w|<\frac{|\lambda|}{\mathrm{a}}\right\}$.

(ii) If $\mathrm{D}_{2}=\left\{z \in \mathbb{C}: \operatorname{Re}(z)<0\right.$ and $\left.\frac{1}{a} \leqslant|z|<\frac{2}{a}\right\}$, then $\mathrm{f}_{\lambda, \mathrm{a}}\left(\mathrm{D}_{2}\right) \subset\left\{w \in \mathbb{C}: \frac{|\lambda|}{\mathrm{a}} \leqslant|w|<\frac{2|\lambda|}{\mathrm{a}}\right\}$.

(iii) If $\mathrm{D}_{3}=\left\{z \in \mathbb{C}: \operatorname{Re}(z)<0\right.$ and $\left.|z| \geqslant \frac{2}{\mathrm{a}}\right\}$, then $\mathrm{f}_{\lambda, \mathrm{a}}\left(\mathrm{D}_{3}\right) \subset\left\{w \in \mathbb{C}:|w|>\frac{|\lambda|}{\mathrm{a}}\right\}$.

Proof. Let $h(z)=\mathrm{e}^{\mathrm{a} z}$ for an arbitrary fixed $z \in \mathrm{H}^{-}$. Suppose that the line segment $\gamma$ is defined by $\gamma(\mathrm{t})=\mathrm{t} z, \mathrm{t} \in[0,1]$. Then, for $\mathrm{a}>0$,

$$
\int_{\gamma} h(z) d z=\int_{0}^{1} h(\gamma(t)) \gamma^{\prime}(t) d t=z \int_{0}^{1} e^{a t z} d t=\frac{1}{a}\left(e^{a z}-1\right) .
$$

Since $m \equiv \min _{t \in[0,1]}|h(\gamma(t))|=\min _{t \in[0,1]}\left|\left(e^{a}\right)^{t z}\right|>0$ for $z \in H^{-}$, by Equation (4.4),

$$
\left|e^{a z}+1\right|=\left|a \int_{\gamma} h(z) d z+2\right| \geqslant a m|z|+2>2>a|z| .
$$

Hence, $\left|\frac{2 z}{e^{a z}+1}\right|<\frac{2}{a}$ for all $|z|<\frac{2}{a}$. Then

$$
\left|f_{\lambda, a}(z)\right|=\left|\lambda \frac{2 z}{e^{a z}+1}\right|<\frac{2|\lambda|}{a} \text { for all }|z|<\frac{2}{a} .
$$


(i) If $0<|z|<\frac{1}{a}$, then it is easily seen that $\left|f_{\lambda, a}(z)\right|=\left|\lambda \frac{2 z}{e^{a z}+1}\right|<\frac{|\lambda|}{a}$. It shows that $f_{\lambda, a}(z)$ maps $H^{-}$ inside the open disk centered at origin and having radius $\frac{|\lambda|}{a}$ for $|z|<\frac{1}{a}$.

(ii) Using Equation (4.5), we can see that $f_{\lambda, a}(z)$ maps $\mathrm{H}^{-}$in the annulus with inner radius $\frac{|\lambda|}{\mathrm{a}}$ including boundary and outer radius $\frac{2|\lambda|}{a}$ without boundary for $\frac{1}{a} \leqslant|z|<\frac{2}{a}$.

(iii) Since $M \equiv \max _{\mathfrak{t} \in[0,1]}|\mathrm{h}(\gamma(\mathrm{t}))|=\max _{\mathrm{t} \in[0,1]}\left|\left(e^{\mathfrak{a}}\right)^{\mathrm{t} z}\right|<1$ for $z \in \mathrm{H}^{-}$, by Equation (4.4),

$$
\left|e^{\mathrm{a} z}+1\right|=\left|\mathrm{a} \int_{\gamma} \mathrm{h}(z) \mathrm{d} z+2\right| \leqslant \mathrm{aM}|z|+2<\mathrm{a}|z|+2<2 \mathrm{a}|z|
$$

so $\left|\frac{2 z}{e^{a z}+1}\right|>\frac{1}{a}$ for all $|z| \geqslant \frac{2}{a}$. Therefore, it follows that $\left|f_{\lambda, a}(z)\right|=\left|\lambda \frac{2 z}{e^{a z}+1}\right|>\frac{|\lambda|}{a}$ for all $|z| \geqslant \frac{2}{a}$. This shows that the function $f_{\lambda, a}(z)$ maps the left half plane $\mathrm{H}^{-}$exterior of the open disk centered at origin and having radius $\frac{|\lambda|}{\mathrm{a}}$ for $|z| \geqslant \frac{2}{\mathrm{a}}$.

Theorem 4.5. Let $\mathrm{f}_{\lambda, \mathrm{a}} \in \mathcal{F}$ with $\mathrm{a}<0$.

(i) If $\mathrm{D}_{1}=\left\{z \in \mathbb{C}: \operatorname{Re}(z)>0\right.$ and $\left.|z|<\frac{1}{|\mathbf{a}|}\right\}$, then $\mathrm{f}_{\lambda, \mathrm{a}}\left(\mathrm{D}_{1}\right) \subset\left\{w \in \mathbb{C}:|w|<\left|\frac{\lambda}{\mathrm{a}}\right|\right\}$.

(ii) If $\mathrm{D}_{2}=\left\{z \in \mathbb{C}: \operatorname{Re}(z)>0\right.$ and $\left.\frac{1}{|\mathbf{a}|} \leqslant|z|<\frac{2}{|\mathbf{a}|}\right\}$, then $\mathrm{f}_{\lambda, \mathrm{a}}\left(\mathrm{D}_{2}\right) \subset\left\{w \in \mathbb{C}:\left|\frac{\lambda}{\mathrm{a}}\right| \leqslant|w|<\frac{2|\lambda|}{|\mathbf{a}|}\right\}$.

(iii) If $\mathrm{D}_{3}=\left\{z \in \mathbb{C}: \operatorname{Re}(z)>0\right.$ and $\left.|z| \geqslant \frac{2}{|\mathrm{a}|}\right\}$, then $\mathrm{f}_{\lambda, \mathrm{a}}\left(\mathrm{D}_{3}\right) \subset\left\{w \in \mathbb{C}:|w|>\frac{|\lambda|}{|\mathrm{a}|}\right\}$.

The proof of this theorem is omitted since it is analogous to Theorem 4.4.

The following theorems give that the critical values of $f_{\lambda, a} \in \mathcal{F}$ lie in the open disk, the annulus and exterior of the open disk according to mapping of three regions.

Theorem 4.6. Let $\mathrm{f}_{\lambda, \mathrm{a}} \in \mathcal{F}$. Then, for $\mathrm{a}>0$, the critical values of $\mathrm{f}_{\lambda, \mathrm{a}}(z)$ lie

(i) inside the open disk centered at origin and having radius $\frac{|\lambda|}{a}$ for $|z|<\frac{1}{a}$;

(ii) in the annulus with inner radius $\frac{|\lambda|}{a}$ including boundary and outer radius $\frac{2|\lambda|}{a}$ without boundary for $\frac{1}{a} \leqslant$ $|z|<\frac{2}{a}$

(iii) exterior of the open disk centered at origin and having radius $\frac{|\lambda|}{a}$ for $|z| \geqslant \frac{2}{a}$.

Proof. By Theorem 4.2, the function $\mathrm{f}_{\lambda, \mathrm{a}}^{\prime}(z)$ has no zeros in the right half plane $\mathrm{H}^{+}$. Hence, all the critical points lie in the left half plane $\mathrm{H}^{-}$. By Theorem $4.4, \mathrm{f}_{\lambda, \mathrm{a}} \in \mathcal{F}$ maps the left half plane $\mathrm{H}^{-}$in the open disk, the annulus and exterior of the open disk. This completes the proof of theorem.

Theorem 4.7. Let $\mathrm{f}_{\lambda, \mathrm{a}} \in \mathcal{F}$. Then, for $\mathrm{a}<0$, the critical values of $\mathrm{f}_{\lambda, \mathrm{a}}(z)$ lie

(i) inside the open disk centered at origin and having radius $\left|\frac{\lambda}{\mathrm{a}}\right|$ for $|z|<\frac{1}{|\mathbf{a}|}$;

(ii) in the annulus with inner radius $\left|\frac{\lambda}{\mathrm{a}}\right|$ including boundary and outer radius $\frac{2|\lambda|}{|\mathrm{a}|}$ without boundary for $\frac{1}{|\mathrm{a}|} \leqslant$ $|z|<\frac{2}{|\mathbf{a}|} ;$

(iii) exterior of the open disk centered at origin and having radius $\frac{|\lambda|}{|\mathbf{a}|}$ for $|z| \geqslant \frac{2}{|\mathbf{a}|}$.

The proof of this theorem can be obtained as similar to Theorem 4.6.

Remark 4.8. The result, all the critical values of functions lie in the left half plane [9, Theorem 2.4], seems incorrect since the left half plane maps inside the open disks and the annulus about the origin with some radius. But these open disks and the annulus must contain the part of the right half plane. 


\section{Conclusion}

In the present paper, we have been generalized the work from one-parameter family of functions $\lambda \frac{2 z}{e^{z}+1}$ to a two-parameter family of functions $\lambda \frac{2 z}{e^{a z}+1}$, which arises from generalized generating function of Apostol-Genocchi numbers. The real fixed points and singular values of this two-parameter family of $f_{\lambda, a}(z)=\lambda \frac{2 z}{e^{a z}+1}, \lambda, a \in \mathbb{R} \backslash\{0\}$ have been investigated. The real fixed points of $f_{\lambda, a}(z)$ as well as their nature have described. In the real dynamics, the bifurcation and chaos have been occurred. It is seen that the function $f_{\lambda, a}(z)$ has infinitely many singular values for both $a>0$ and $a<0$. The critical values of $f_{\lambda, a}(z)$ lie inside the open disk, the annulus and exterior of the open disk for both $a>0$ and $a<0$.

\section{Acknowledgment}

The author is thankful to referees for providing helpful suggestions to improve this manuscript.

\section{References}

[1] M. Akbari, M. Rabii, Real cubic polynomials with a fixed point of multiplicity two, Indag. Math. (N.S.), 26 (2015), 64-74. 1

[2] X.-H. Hua, C.-C. Yang, Dynamics of Transcendental Functions, Gordon and Breach Science Publishers, Amsterdam, (1998). 1

[3] C. M. Jiang, S. T. Liu, D. Wang, Generalized Combination Complex Synchronization for Fractional-Order Chaotic Complex Systems, Entropy, 17 (2015), 5199-5217. 1

[4] C. M. Jiang, F. F. Zhang, T. X. Li, Synchronization and antisynchronization of N-coupled fractional-order complex chaotic systems with ring connection, Math. Method. Appl. Sci., 41 (2018), 2625-2638. 1

[5] H. Jolany, H. Sharifi, R. E. Alikelaye, Some results for the Apostol-Genocchi polynomials of higher order, Bull. Malays. Math. Sci. Soc. (2), 36 (2013), 465-479. 1

[6] G. P. Kapoor, M. G. P. Prasad, Dynamics of $\left(e^{z}-1\right) / z$ : the Julia set and bifurcation, Ergod. Th. Dynam. Sys., 18 (1998), 1363-1383. 1, 4

[7] R. Kautz, Chaos-the science of predictable random motion, Oxford University Press, Oxford, (2011). 1

[8] M. G. Lee, C. C. Ho, Fixed points of two-parameter family of function $\lambda\left(\frac{x}{b^{x}-1}\right)^{n}$, Applied Mathematics, 6 (2015), 576-584. 1

[9] D. K. Lim, Fixed points and dynamics on generating function of Genocchi numbers, J. Nonlinear Sci. Appl., 9 (2016), 933-939. 1, 2.2, 4.8

[10] A. A. Magrenán, J. M. Gutiérrez, Real dynamics for damped Newtons method applied to cubic polynomials, J. Comput. Appl. Math., 275 (2015), 527-538. 1

[11] T. Nayak, M. G. P. Prasad, Iteration of certain meromorphic functions with unbounded singular values, Ergodic Theory Dynam. Systems, 30 (2010), 877-891. 1

[12] T. Nayak, M. G. P. Prasad, Julia sets of Joukowski-Exponential maps, Complex Anal. Oper. Theory, 8 (2014), 1061-1076. 1

[13] M. G. P. Prasad, Chaotic burst in the dynamics of $\mathrm{f}_{\lambda}(z)=\lambda \frac{\sinh (z)}{z}$, Regul. Chaotic Dyn., 10 (2005), 71-80. 1

[14] M. G. P. Prasad, T. Nayak, Dynamics of certain class of critically bounded entire transcendental functions, J. Math. Anal. Appl., 329 (2007), 1446-1459. 1

[15] A. G. Radwan, On some generalized discrete logistic maps, J. Adv. Res., 4 (2013), 163-171. 1

[16] M. Sajid, Real and complex dynamics of one parameter family of meromorphic functions, Far East J. Dyn. Syst., 19 (2012), 89-105. 1

[17] M. Sajid, On real fixed points of one parameter family of function $\frac{x}{b^{x}-1}$, Tamkang J. Math., 46 (2015), 61-65. 1

[18] M. Sajid, Singular values and fixed points of family of generating function of Bernoulli's numbers, J. Nonlinear Sci. Appl., 8 (2015), 17-22. 1

[19] M. Sajid, Singular Values of One Parameter Family $\lambda \frac{\mathrm{b}^{z}-1}{z}$, J. Math. Comput. Sci., 15 (2015), 204-208. 1

[20] M. Sajid, Singular values of one parameter family of generalized generating function of Bernoulli's numbers, Appl. Math. Inf. Sci., 9 (2015), 2921-2924. 1

[21] M. Sajid, A. S. Alsuwaiyan, Chaotic behavior in the real dynamics of a one parameter family of functions, Int. J. Appl. Sci. Eng., 12 (2014), 283-301. 1, 1

[22] M. Sajid, G. P. Kapoor, Dynamics of a family of non-critically finite even transcendental meromorphic functions, Regul. Chaotic Dyn., 9 (2004), 143-162. 1

[23] M. Sajid, G. P. Kapoor, Dynamics of transcendental meromorphic functions $(z+\mu) e^{z} /(z+\mu+4)$ having rational Schwarzian derivative, Dynam. Syst., 22 (2007), 323-337. 1

[24] F.-F. Zhang, S.-T. Liu, W.-Y. Yu, Modified projective synchronization with complex scaling factors of uncertain real chaos and complex chaos, Chinese Physics B, 22 (2013), 141-151. 1

[25] J. H. Zheng, On fixed-points and singular values of transcendental meromorphic functions, Sci. China Math., 53 (2010), 887-894. 1 